\title{
Sodium Flux during Haemodialysis
}

\author{
B.S. TILLEY ${ }^{1} \dagger$ \\ ${ }^{1}$ Worcester Polytechnic Institute, USA
}

(Communicated to MIIR on 6 April 2021)

Study Group: Canadian Biomedical Problem Solving Workshop. 22-26 June 2009, Toronto

Communicated by: Huaxiong Huang

Industrial Partner: Brigham and Women's Hospital, Boston, MA, USA and Harvard Medical School

Presenter: Sushrat Waikar (Brigham and Women's Hospital, Boston, MA, and Harvard Medical School).

Team Members: C. Sean Bohun, UOIT; Chris Breward, Oxford; Radina Droumeva, SFU; Ian Griffiths, Oxford; Matt Hennessy, UOIT; Huaxiong Huang, York University; Matt Kloosterman, UOIT; Adam Pan, Toronto; Chris Raymond; Delaware; Don Schwendeman, RPI; Jennifer Siggers, Imperial College London; B.S. Tilley, WPI; Marcus Tindall, University of Reading; Jonathan Wattis, University of Nottingham; Jonathan Whiteley, Oxford; Robert Whittaker, Oxford; J.F. Williams, SFU.

Industrial Sector: Biomedical and Healthcare

Tools: Advection, Fluid Dynamics, Solute Transport, Electrokinetics, Osmosis

Key Words: Dialysis Modelling, Electrolyte

MSC2020 Codes: 76

$\dagger$ tilley@wpi.edu 


\section{Summary}

Dialysis is aimed at the removal of charged ionic species in the blood that arise from complications in kidney disease. Although the process for the removal of urea and other unwanted charged species is understood, the effect of this removal on the net sodium concentration in the blood after treatment is not clear. In this report, we focus on formulating a fundamentally-based model to address this question. We consider the formulation near the membrane at the pore scale in order to determine effective jump conditions in ionic concentrations, electric potential and flow rate based on the membrane properties, and in order to determine whether electroneutrality holds within the pore. Secondly, we consider the local blood-cell concentration within one of the fibres and how this varies axially within the dialysis cartridge. Lastly, we consider a simple one-dimensional model of the charged species problem and find that advection transport through the membrane is important for sodium transport, but less pertinent for transport of other cation species. 


\section{Introduction}

Kidney disease is treated worldwide by the use of surgical transplantation and more often, due to lack of donor organs, dialysis. The overall aim of dialysis is to remove waste products, such as urea, from the patient's blood. Dialysis is performed using a dilayzer machine, whereby blood is taken from the patient, and passed through the dialyzer where filtration occurs, before being returned to the patients bloodstream in a continual process. A single dialysis session can last anywhere up to four hours.

The process, however, is by no means perfect. Patients post-dialysis show a wide variation in the concentration of certain solutes within their blood, for instance sodium. Sodium imbalance, for instance excess, can lead to higher water retention which can have adverse health effects. With end stage renal disease increasing by between $5 \%$ and $10 \%$ per year worldwide, there is an ever increasing need to more fully understand and improve the dialysis process. In this report we present a number of mathematical models which go some way towards providing a deterministic model of dialysis by focusing on the mechanisms involved in the filtration of waste products from the blood in a dialyzer.

A typical dialyzer is illustrated in Figure 1. The machine works by passing blood through perforated tubes encased in a cassette within the dialyzer. As blood flows in one direction down the tubes (radius $200 \mu \mathrm{m}$ ), dialysate, the filtration fluid, is passed in the opposite direction, thus forming a counter-current flow regime between the two fluids. The two flows are connected by a number of smaller channels (radius $5 \mu \mathrm{m}$ ), which allows exchange of solutes between the blood and dialysate. The exchange of solutes is driven by both diffusion and convection through the channels. Details on the geometry of the tubes, the size of the interconnecting channels, channels per tube and other details relevant to the problems discussed in this report are detailed in Table 1.

Dialysate is designed to filter the blood during the dialysis process (which can take anywhere up to four hours) without altering the solute concentrations within it. As such the dialysate contains the main solutes found within blood (e.g. potassium- $\mathrm{K}^{+}$, sodium$\mathrm{Na}^{+}$, and chlorine- $\mathrm{Cl}^{-}$). The unfiltered blood contains these same solutes as well as urea (to be filtered), along with blood cells and various negatively-charged proteins that are not filtered.

Whilst it is relatively simple to understand the basic elements of dialysis, the effect of the physical processes (fluid flow, solute transport) within a dialyzer requires detailed quantitative understanding of each process in order to accurately understand the overall blood filtration effects. As such the problem is ripe for mathematical modelling, with a number of models formulated to date. We briefly review here the different modelling approaches. For further details the reader should consult the recent review of [3].

Mathematical modelling approaches fall in to two main areas: (i) compartmental ordinary differential equation (ODE) which model the patient-dialyzer system; and (ii) models which describe the spatial variation in solute concentration fluid flow within a dialyzer using the theory of partial differential equations (PDEs). Both model types are generally parameterised by comparing and/or fitting model outcomes to experimental and/or patient data. Their main use then is in predicting the effective removal of urea from the blood stream and the solute concentration at the end of the process. Sodium is a common solute of interest given the issue of overhydration and the resultant health issues.

Compartmental models include descriptions of the fluid (blood plasma, dialysate) and solutes within the patient/dialyzer system, and focus on key issues, for instance urea or 


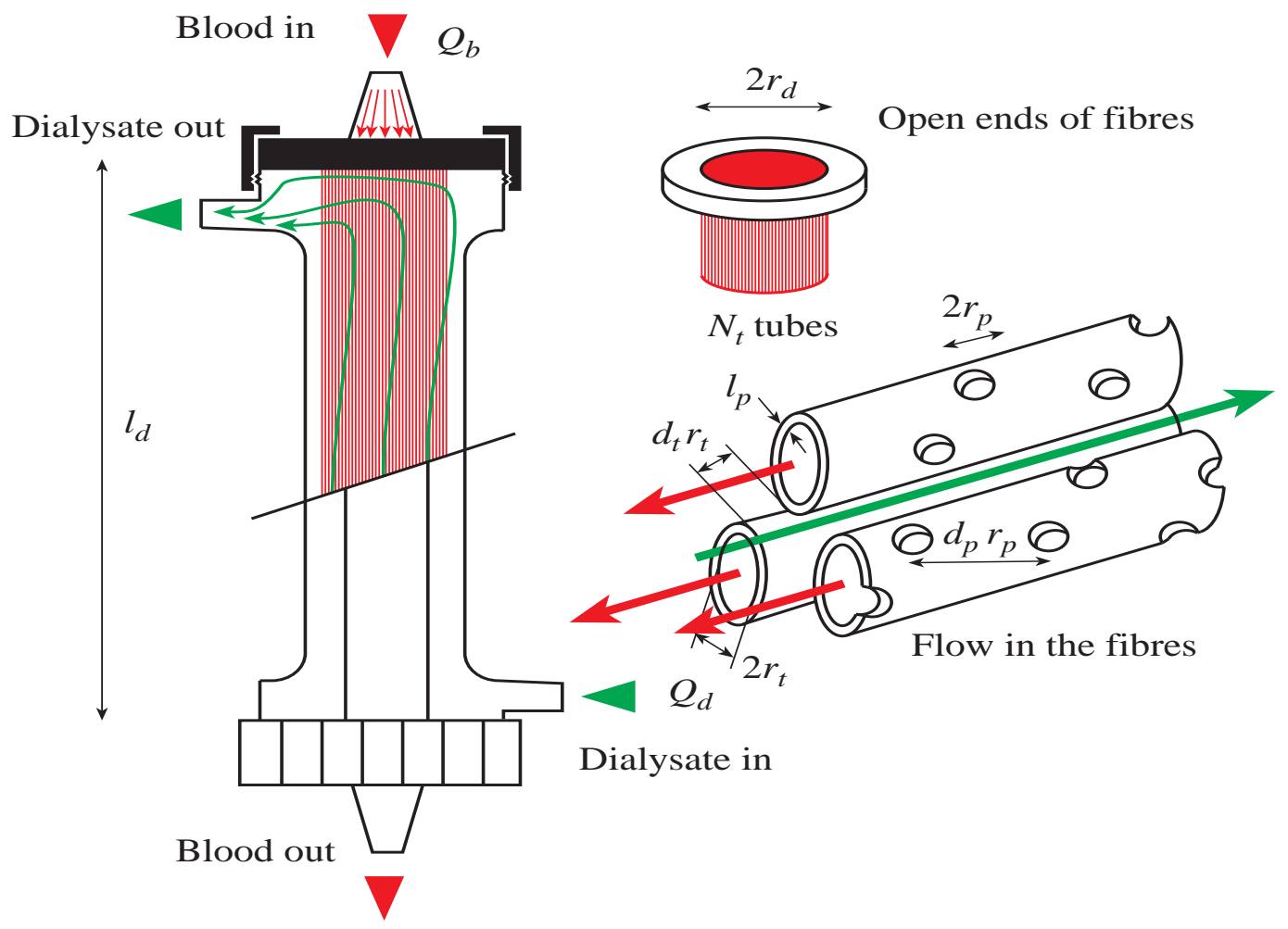

Figure 1 A schematic representation of a typical dialyzer (left) and the geometrical layout of a cassette showing the porosity of the tubes (right).

specific solute clearance. The basic urea model [3] considers a single compartment into which urea passes and is then separated into a fluid of volume $V$ and urea of concentration $C$. Urea is then removed from the compartment at a constant rate. This leads to a single ODE which can be solved analytically to determine the change in urea concetration over time. More complicated models consider different compartmentalisations. For instance [9] built on the work of [10] and considered a three compartment model: intracellular (representing fluid inside patient cells), interstitial (representing fluid outside cells) separating the cell from the final compartment, the blood plasma. The dialysate only affects the blood plasma. Each compartment is separated by a 'membrane'. In the intracellular to extracellular compartments this is equivalent to that of the cell membrane, whereas the interstitial to blood plasma membrane represents resistance of solute flow between these regions. Similar models have been used to fit to experimental data regarding the profile of solutes from patients and experimentation on dialyzers [6].

A similar model [12] used three compartments for the body fluids (plasma, interstitial and intracellular, as mentioned above) and tracked transport of the main solutes between these compartments and between the blood and dialysate. The authors claim that the required parameters for the model can be determined a priori based on the body weight and measured pre-dialysis plasma concentration values for patients, and that their model's predictions agreed fairly well with the results from multiple dialysis sessions for six patients. They suggest using the model to tailor dialysis sessions (i.e. by varying the length of the dialysis session and/or the concentrations of various ions in the dialysate solution) 
to the needs of individual patients. The desirability of implementing such a scheme, and some of the difficulties of doing so in an actual clinical setting, are discussed in [14]. Profiled hemodialysis is an even more ambitious scheme for minimizing the unpleasant side effects of dialysis by varying operational parameters of the dialysis machine (e.g. sodium concentration in dialysate) as a function of time [9].

In [13], some of the issues involved in building a compartmental model of the dialysis process are discussed. Starting from a simple one compartment model, several more detailed models with successively more compartments are built and investigated. All of the models considered in this reference only track one concentration, that of the main 'toxic substance' (presumably urea) that the dialysis treatment is designed to remove from the blood.

There are considerably fewer models of dialysis which include descriptions of the variation in fluid flow and solute concentrations within a dialyzer (see the introduction to [5] for a brief review). Such models are generally solved numerically. Ding et al. [4] modelled a hollow fiber hemodialyzer as two interpenetrating porous regions, of differing porosity, containing blood and dialysate respectively. The two regions are separated by a thin porous membrane. The fluid flow of blood and dialysate were considered to be governed by the Navier-Stokes equations, with the concentration of dialysate and blood modelled by quasisteady state diffusion-convection equations in each region. The membrane flow is described by a difference in the pressure between the blood and dialysate regions and the concentration of the blood and dialysate. Ding et al. [4] obtained numerical solutions to their system of equations to predict the spatial variation in urea along the length of the dialyzer. This was compared with experimental data and shown to be in good agreement. Similar porous media models have been formulated and solved by Nordon and Shindhelm [7] and Osuga et al. [8].

Of interest here is to understand the dominant mechanisms of solute transport from the blood to the dialysate under normal operating conditions. The pore-scale processes within the membrane, which include advection from the flow of liquid from the blood to the dialyzer, electrodiffusion effects due to the induced electric fields from the ions themselves, and reverse osmosis effects due to the concentration jump across the membrane, are key to understanding this transport. Inherent in this understanding is the characteristic lengths and other scales related to this problem. In Table 1, we list the characteristic geometric parameters of the cartridge, the tubing, and the typical pore scales of the semi-permeable membrane, and we list the diffusion and concentration values in Table 1 . In Table 1 we list the effective flow values during normal operating conditions, along with some typical nondimensional quantities based on the values in Table 1. Since the dialysate and the blood contains concentrations of charged species, we list some characteristic electrical parameters in Table 1 ,

In this report, we focus on three distinct problems. The first problem relates to the dependence of the solvent and solute transport through the pores in the semi-permeable membrane (see Section 2). The second problem, discussed in Section 3, considers the plasma/fluid transport through the membrane due to pressure differences in the blood and dialysate regions. Finally, in Section 4, we consider a simple one-dimensional model of solute transport across the membrane. We conclude in Section 5.

\section{The problem in a single pore}

The dialysis process of removing fluid, urea and potassium ions from the patient's plasma is controlled by the transport properties of the membrane. To understand these 
Table 1 Geometrical quantities from literature, many from [17].

\begin{tabular}{|l|l|l|}
\hline Quantity & Symbol & Value \\
\hline Dialyser length & $l_{d}$ & $0.2 \mathrm{~m}$ \\
Dialyser radius & $r_{d}$ & $0.02 \mathrm{~m}$ \\
Number of tubes & $N_{t}$ & 12,000 \\
Tube radius & $r_{t}$ & $10^{-4} \mathrm{~m}$ \\
Number of pores per unit area & $n_{p p u a}$ & $10^{13} \mathrm{~m}^{-2}$ \\
Length of pore (wall thickness) & $l_{p}$ & $15 \times 10^{-6} \mathrm{~m}$ \\
Pore radius & $r_{p}$ & $5 \times 10^{-9} \mathrm{~m}$ \\
Cross-sectional area of dialyser & $A_{d}=\pi r_{d}^{2}$ & $1.3 \times 10^{-3} \mathrm{~m}^{2}$ \\
Inner area - single tube & $A_{t i}=\pi r_{t}^{2}$ & $3.1 \times 10^{-8} \mathrm{~m}^{2}$ \\
Outer area - single tube & $A_{t o}=\pi\left(r_{t}+l_{p}\right)^{2}$ & $4.2 \times 10^{-8} \mathrm{~m}^{2}$ \\
Area of inner tube surface & $A_{s}=2 N_{t} \pi r_{t} l_{d}$ & $1.5 \mathrm{~m}^{2}$ \\
Fraction of cross-sectional area & $\phi_{t}=N_{t} A_{t o} / A_{d}$ & 0.40 \\
$\quad$ occupied by tubes & $d_{t}=\sqrt{2 \pi /\left(\sqrt{3} \phi_{t}\right)}-2$ & 1.0 \\
Scaled distance between neighbouring tubes & & \\
(assumes triangular lattice) & $N_{p}=2 N_{t} \pi r_{t} l_{d} n_{p p u a}$ & $1.5 \times 10^{13}$ \\
Number of pores & $A_{p}=\pi r_{p}^{2}$ & $7.9 \times 10^{-17} \mathrm{~m}^{2}$ \\
Area of pore & $\phi_{p}=\pi r_{p}^{2} n_{p p u a}$ & $7.9 \times 10^{-4}$ \\
Pore fraction of tube surface area & $d_{p}=\sqrt{2 \pi /\left(\sqrt{3} \phi_{p}\right)}-2$ & 66 \\
Nondim distance between neighbouring pores & \\
(assumes triangular lattice) & & \\
\hline
\end{tabular}

Table 2 Flux/flow quantities.

\begin{tabular}{|l|l|l|}
\hline Quantity & Symbol & Value \\
\hline Fluxes: & & \\
\hline Flux of blood & $Q_{b}$ & $400 \times 10^{-6} / 60 \mathrm{~m}^{3} / \mathrm{s}$ \\
Flux of dialysate & $Q_{d}$ & $800 \times 10^{-6} / 60 \mathrm{~m}^{3} / \mathrm{s}$ \\
Blood filtration rate & $Q_{p}$ & $10^{-3} / 3600 \mathrm{~m}^{3} / \mathrm{s}$ \\
\hline Flow: & & \\
\hline Average blood velocity & $U_{b}=Q_{b} /\left(N_{t} A_{t i}\right)$ & $0.0018 \mathrm{~m} / \mathrm{s}$ \\
Average diastolate velocity & $U_{d}=Q_{d} /\left(A_{d}\left(1-\phi_{t}\right)\right)$ & $0.0018 \mathrm{~m} / \mathrm{s}$ \\
Average velocity in pore & $U_{p}=Q_{p} /\left(N_{p} A_{p}\right)$ & $2.3 \times 10^{-4} \mathrm{~m} / \mathrm{s}$ \\
Reynolds number of the blood & $R e_{b}=2 U_{b} r_{t} / \nu_{b}$ & 0.88 \\
Reynolds number of the diastolate & $R e_{d}=U_{d} r_{t} d_{t} / \nu_{d}$ & 0.45 \\
Péclet number of sodium & & \\
$\quad$ (or potassium) in blood & $P e_{N a b}=2 U_{b} r_{d} / D_{N a}$ & $3.5 \times 10^{5}$ \\
Péclet number of sodium & & \\
$\quad$ (or potassium) in pore & $P e_{N a p}=U_{p} l_{p} / D_{N a}$ & 1.8 \\
\hline
\end{tabular}

properties fundamentally, we need to investigate the ion and fluid transport through a single pore. During the workshop, we considered only transport of the ions by a prescribed pressure field, which is described in Section 2.1 below. In general, however, the flow in these membranes is driven by concentration gradients local to the membrane in the bulk. 
Table 3 Electrical quantities from literature, many from [17].

\begin{tabular}{|l|l|l|}
\hline Quantity & Symbol & Value \\
\hline Electric constants: & & \\
\hline Proton charge & $e$ & $1.602 \times 10^{-19} \mathrm{C}$ \\
Permittivity of free space & $\epsilon_{0}$ & $8.85 \times 10^{-12} \mathrm{~A}^{2} \mathrm{~s}^{4} \mathrm{~kg}^{-1} \mathrm{~m}^{-3}$ \\
Relative permittivity & $\epsilon_{R}$ & 80 \\
Boltzmann's constant & $k_{B}$ & $1.38 \times 10^{-23} \mathrm{~J} / \mathrm{K}$ \\
Faraday's constant & $F=N_{a} e$ & $9.4 \times 10^{4} \mathrm{C} / \mathrm{mol}$ \\
Debye lengthscale & $\sqrt{\epsilon_{0} \epsilon_{R} k_{B} T /\left(4 \pi e F c_{N a}\right)}$ & $1.2 \times 10^{-9} \mathrm{~m}$ \\
\hline
\end{tabular}

Table 4 Diffusion and viscosity quantities from literature, many from [17].

\begin{tabular}{|l|l|l|}
\hline Quantity & Symbol & Value \\
\hline Diffusion and viscosity: & & \\
\hline Kinematic viscosity of blood & $\nu_{b}$ & $4 \times 10^{-6} \mathrm{~m}^{2} / \mathrm{s}$ \\
Kinematic viscosity of dialysate & $\nu_{d}$ & $4 \times 10^{-6} \mathrm{~m}^{2} / \mathrm{s}$ \\
Diffusion coefficient of sodium & $D_{N a}$ & $2 \times 10^{-9} \mathrm{~m}^{2} / \mathrm{s}$ \\
Diffusion coefficient of potassium & $D_{K}$ & $2 \times 10^{-9} \mathrm{~m}^{2} / \mathrm{s}$ \\
Diffusion coefficient of urea & $D_{\text {urea }}$ & $1.8 \times 10^{-9} \mathrm{~m}^{2} / \mathrm{s}$ \\
Operating Temperature & $T$ & $300 \mathrm{~K}$ \\
Avogadro's number & $N_{a}$ & $6.023 \times 10^{23}$ \\
Sodium concentration in blood & $c_{N a}$ & $140 \mathrm{mmol} / \mathrm{l}=1.4 \times 10^{2} \mathrm{~mol} / \mathrm{m}^{3}$ \\
\hline
\end{tabular}

Table 5 Typical pressure drops measured in the cartridge during dialysis [15].

\begin{tabular}{|l|c|}
\hline Pressure drop & Value in literature $(\mathrm{Pa})$ \\
\hline Blood along tubes & 2,666 \\
Dialysate & 6,666 \\
Across pores $\left(\Delta p_{p}\right)$ & $40,000-49,400$ \\
\hline
\end{tabular}

We perform an analysis by Anderson and Malone [2], extended to electrolytes, to include osmotic effects. In this case, the flow field and concentration fields are necessarily coupled. This work is found in Section 2.2. Simple examples of these field equations are presented in each section.

The rationale for this extension is based on the following argument. Fournier [15] gives the blood and dialysate gauge pressures as 117,300 $\mathrm{Pa}$ and 74,600 $\mathrm{Pa}$ respectively and the pressure drops as 2,666 $\mathrm{Pa}$ and $6,666 \mathrm{~Pa}$ respectively, meaning the pressure drop across the pores varies between 40,000 $\mathrm{Pa}$ and 49,400 $\mathrm{Pa}$. These values are compared in Table 2 . This shows that the comparison is quite bad, particularly in the pores. However, both the calculated and the actual values do suggest that the pressure drop across the pores is much larger than that along the length of the dialyzer. We may also calculate the permeability (volume flux per unit area per unit pressure drop) across the walls of the tubes, which for $\Delta p_{p}=45 \mathrm{kPa}$ gives

$$
\frac{Q p}{2 \pi r_{t} l_{d} N_{t} \Delta p_{p}} \approx 4.1 \times 10^{-12} \mathrm{~m}^{2} \mathrm{~s} / \mathrm{kg}
$$




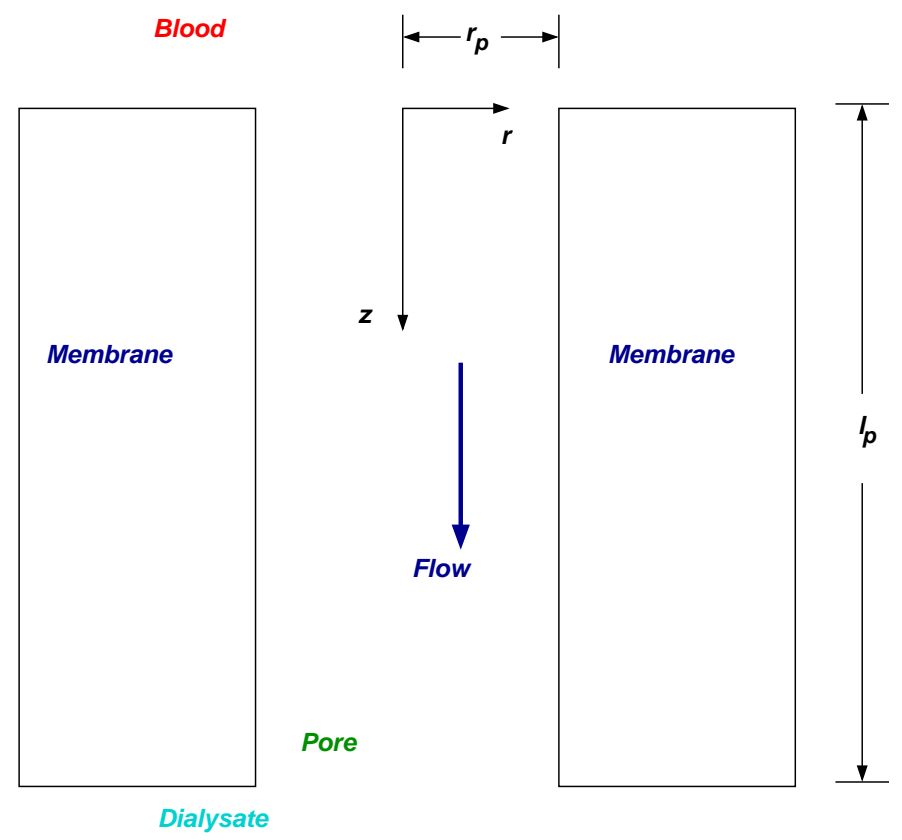

Figure 2 Geometry of pore problem investigated in Section 2.

Values of the permeability of biomaterials are given as $10^{-14}-10^{-9} \mathrm{~m}^{2} \mathrm{~s} / \mathrm{kg}$, and in dialyzers are between $2 \times 10^{-12}$ and $4 \times 10^{-11} \mathrm{~m}^{2} \mathrm{~s} / \mathrm{kg}$, so these values are consistent with purely pressure-driven flow.

The geometry of the pore problem is shown in Figure 2. The membrane surface on the blood side is at $z=0$, and we focus on one pore of radius $r_{p}$ and length $l_{p}$.

2.1 Pressure-driven flow. The governing equations for the axisymmetric ion transport are given by [16]

$$
\begin{aligned}
\frac{\partial c_{i}}{\partial t}+\mathbf{u} \cdot \nabla c_{i} & =\nabla \cdot\left(D_{i} \boldsymbol{\nabla} c_{i}\right)+\nabla \cdot\left(c_{i} z_{i} k_{i} e \boldsymbol{\nabla} \phi\right), \\
\epsilon \nabla^{2} \phi & =-4 \pi \sum_{i} F c_{i} z_{i}, \\
w & =\frac{1}{2} w_{0}\left(1-\frac{r^{2}}{r_{p}^{2}}\right),
\end{aligned}
$$

where $\mathbf{u}=u \mathbf{r}+w \mathbf{z}, \phi$ is the electric potential, $F$ is Faraday's constant (see Tables 1 and 1 ), and (2.2)-(2.3) apply on an axisymmtric domain $0<r<r_{p}$ and $0<z<l_{p}$. Equation (2.2) represents the transport of species $i$ through the pore, where $i=1,2, \ldots N$ denote distinct species, and where $c_{i}$ is the molar concentration of species $i$. The second term on the left-hand side represents advection of charge through the pore. The two terms on the right-hand side of (2.2) (from left to right) represent diffusion of charge through the pore and electrodiffusion, respectively. Each species in general has its own rate of diffusion, $D_{i}=k_{B} T k_{i}$, where $k_{B}$ is Boltzmann's constant, $T$ is the temperature of the solution, $k_{i}$ is species mobility. In addition, each $i$-ion has a net charge $z_{i} e$, where $e$ is the fundamental charge of an electron (see Table 1 for specific values). We assume the flow of the solute 
within the pore is pressure-driven Stokes flow in cylindrical coordinates, which results in the quadratic profile shown in (2.4).

Electrodiffusion is driven by gradients in the electric potential $\phi$. This potenial is found through (2.3), which represents Gauss's law in electrostatics, where $\epsilon$ is the relative dielectric permittivity of the solvent. Note that the net charge density $\rho=\sum_{i} e F z_{i} c_{i}$ is the quantity that drives potential gradients.

Boundary conditions for this problem are no flux conditions at $r \rightarrow 0$ and $r=r_{p}$,

$$
\begin{array}{ll}
r \rightarrow 0: & r \frac{\partial c_{i}}{\partial r} \rightarrow 0, r \frac{\partial \phi}{\partial r} \rightarrow 0, \\
r=r_{p}: & \frac{\partial c_{i}}{\partial r}=\frac{\partial \phi}{\partial r}=0 .
\end{array}
$$

We are interested in finding effective jump conditions from above and below the pore, and so we assume Dirichlet boundary conditions at $z=0$ and $z=l_{p}$

$$
\begin{aligned}
z=0: & c_{i}=C_{i}^{(+)}, \phi=\Phi^{(+)}, \\
z=l_{p}: & c_{i}=C_{i}^{(-)}, \phi=\Phi^{(-)} .
\end{aligned}
$$

We scale $r$ on $r_{p}, z$ on $l_{p}, t$ on $l_{p} / w_{o}, \mathbf{u}$ on $w_{o}, c_{i}$ on $C_{o}$, and $\phi$ on $k_{B} T / e$ to arrive at the following nondimensional problem

$$
\begin{aligned}
\delta^{2} P e_{i}\left(\frac{\partial C_{i}}{\partial t}+\frac{1}{2}\left(1-r^{2}\right) \frac{\partial C_{i}}{\partial z}\right)= & \frac{1}{r} \frac{\partial}{\partial r}\left(r \frac{\partial C_{i}}{\partial r}\right)+\delta^{2} \frac{\partial^{2} C_{i}}{\partial z^{2}}+\frac{1}{r} \frac{\partial}{\partial r}\left(z_{i} C_{i} \frac{\partial \phi}{\partial r}\right) \\
& +\delta^{2} \frac{\partial}{\partial z}\left(z_{i} C_{i} \frac{\partial \phi}{\partial z}\right), \\
\frac{1}{r} \frac{\partial}{\partial r}\left(r \frac{\partial \phi}{\partial r}\right)+\delta^{2} \frac{\partial^{2} \phi}{\partial z^{2}}= & -\frac{1}{\lambda^{2}} \sum_{i} z_{i} C_{i},
\end{aligned}
$$

where $\delta=r_{p} / l_{p} \simeq 3 \times 10^{-4}$ is the aspect ratio of the pore, $P e_{i}=w_{o} l_{p} / D_{i} \approx 1.8$ is the Péclet number for each species, and $\lambda=\lambda_{D} / r_{p}$ is the Debye length ratio, where the Debye length is given by $\lambda_{D}=\sqrt{\left(\epsilon k_{B} T\right) /\left(4 \pi e F C_{o}\right)}$. The boundary conditions (2.5)-(2.8) have the same form with this scaling

$$
\begin{aligned}
r \rightarrow 0: & r \frac{\partial c_{i}}{\partial r} \rightarrow 0, r \frac{\partial \phi}{\partial r} \rightarrow 0, \\
r=1: & \frac{\partial c_{i}}{\partial r}=\frac{\partial \phi}{\partial r}=0, \\
z=0: & c_{i}=C_{i}^{(+)}, \phi=\Phi^{(+)}, \\
z=1: & c_{i}=C_{i}^{(-)}, \phi=\Phi^{(-)} .
\end{aligned}
$$

Although the Debye length scale is on the same order of magnitude as the pore radius, we can consider the problem in the limit of small aspect ratio $\delta \rightarrow 0$. We use a regular asymptotic expansion for each of the quantities

$$
C_{i}=n_{i 0}+\delta^{2} n_{i 2}+\ldots, \quad \phi=\phi_{0}+\delta^{2} \phi_{2}+\ldots .
$$


At leading order, we find that

$$
\begin{gathered}
\frac{1}{r} \frac{\partial}{\partial r}\left(r \frac{\partial n_{i 0}}{\partial r}\right)+\frac{1}{r} \frac{\partial}{\partial r}\left(r z_{i} n_{i 0} \frac{\partial \phi_{0}}{\partial r}\right)=0, \\
\frac{1}{r} \frac{\partial}{\partial r}\left(r \frac{\partial \phi_{0}}{\partial r}\right)=-\frac{1}{\lambda^{2}} \sum z_{i} n_{i 0},
\end{gathered}
$$

subject to the no-flux boundary conditions in $r$. We can integrate (2.16) directly in terms of $r$, and note that

$$
\frac{\partial n_{i 0}}{\partial r}+z_{i} n_{i 0} \frac{\partial \phi_{o}}{\partial r}=0,
$$

since the fluxes are zero at $r=0,1$ for all $0<z<1$. A second integration of (2.18) gives the Nerst relation between concentration and potential

$$
n_{i 0}(r, z, t)=A_{i}(z, t) e^{-z_{i} \phi_{o}(r, z, t)} .
$$

Hence, the leading order problem to consider for the potential problem gives

$$
\frac{1}{r} \frac{\partial}{\partial r}\left(r \frac{\partial \phi_{o}}{\partial r}\right)=-\frac{1}{\lambda^{2}} \sum_{i} z_{i} A_{i}(z, t) e^{-z_{i} \phi_{o}}
$$

subject to no-flux boundary conditions (2.11)-(2.12). Note that the trivial solution $\partial \phi_{o} / \partial r=$ 0 , or for $\phi$ independent of $r$ gives the electro-neutrality constraint.

At this stage, we must make a choice in order to solve for (2.20). The simplest choice is to prescribe electro-neutrality ab initio,

$$
\sum_{i} z_{i} n_{i 0}=\sum_{i} z_{i} A_{i}(z, t) e^{-z_{i} \phi_{o}}=0, \quad 0<r<1,
$$

which gives that $\phi_{o}=\phi_{o}(z, t)$ to leading order, and

$$
n_{i 0}(z, t)=A_{i}(z, t) e^{-z_{i} \phi_{o}(z, t)} .
$$

To find the values of $n_{i 0}, \phi_{o}$, we go to the $O\left(\delta^{2}\right)$ problem

$$
\begin{aligned}
P e_{i}\left[\frac{\partial n_{i 0}}{\partial t}+\frac{1}{2}\left(1-r^{2}\right) \frac{\partial n_{i 0}}{\partial z}\right]= & \frac{1}{r} \frac{\partial}{\partial r}\left(r \frac{\partial n_{i 1}}{\partial r}\right)+\frac{\partial^{2} n_{i 0}}{\partial z^{2}}+\frac{1}{r} \frac{\partial}{\partial r}\left(z_{i} n_{i 0} \frac{\partial \phi_{1}}{\partial r}\right) \\
& +\frac{\partial}{\partial z}\left(z_{i} n_{i 0} \frac{\partial \phi_{o}}{\partial z}\right), \\
\frac{1}{r} \frac{\partial}{\partial r}\left(r \frac{\partial \phi_{1}}{\partial r}\right)+\frac{\partial^{2} \phi_{o}}{\partial z^{2}}= & -\frac{1}{\lambda^{2}} \sum_{i} z_{i} n_{i 1},
\end{aligned}
$$

subject to the no-flux boundary conditions (2.11)-(2.12). With these conditions, we can find the effective equation for each $n_{i 0}$ as a function of $\phi_{o}$,

$$
P e_{i}\left(\frac{\partial n_{i 0}}{\partial t}+\frac{1}{4} \frac{\partial n_{i 0}}{\partial z}\right)=\frac{\partial^{2} n_{i 0}}{\partial z^{2}}+\frac{\partial}{\partial z}\left\{z_{i} n_{i 0} \frac{\partial \phi_{o}}{\partial z}\right\} .
$$

The case of monovalent ions is a classical derivation if the Peclet numbers are the same $[1,16]$. If $z_{i}= \pm 1$ and $P e_{i}=P e$, then we can define a conductivity $\sigma$ and a charge density $\rho_{E}$ as

$$
\sigma=\sum_{i} z_{i}^{2} n_{i 0}, \quad \rho_{E}=\sum_{i} z_{i} n_{i 0}=0
$$


By electro-neutrality, the charge density is zero, and we can add all of the equations in (2.24) to obtain

$$
P e\left(\frac{\partial \sigma}{\partial t}+\frac{1}{4} \frac{\partial \sigma}{\partial z}\right)=\frac{\partial^{2} \sigma}{\partial z^{2}}
$$

while multiplying each (2.24) by $z_{i}$ and adding the remainder of the equations gives the relation for the voltage potential

$$
\frac{\partial}{\partial z}\left(\sigma \frac{\partial \phi_{o}}{\partial z}\right)=0
$$

which states that the current density is uniform in the axial direction of the pore. If we prescribe the conductivity along the blood side of the membrane $\sigma=\sigma_{b}$ at $z=0$ and at the dialysate side of the membrane $\sigma=\sigma_{d}$ at $z=1$, then we can analytically find the local conductivity and electric potential in the pore

$$
\begin{aligned}
\sigma & =\frac{e^{P e}-e^{P e z}}{e^{P e}-1} \sigma_{b}+\frac{e^{P e z}-1}{e^{P e}-1} \sigma_{d}, \\
\phi_{0} & =J_{E} \frac{e^{P e}-1}{\sigma_{b} e^{P e}-\sigma_{d}}\left(z-\frac{1}{P e} \log \sigma\right)+\zeta .
\end{aligned}
$$

With (2.27) and (2.28) we can find relations for the net conductivity flux through the pore, along with the net jump in voltage potential

$$
Q_{\sigma}=P e \sigma-\frac{\partial \sigma}{\partial z}=P e \frac{e^{P e} \sigma_{b}-\sigma_{d}}{e^{P e}-1},\left.\quad \phi_{0}\right|_{z=0} ^{z=1}=J_{E} \frac{e^{P e}-1}{e^{P e} \sigma_{b}-\sigma_{d}}\left\{\frac{1}{P e} \log \left[\frac{\sigma_{d}}{\sigma_{b}}\right]+1\right\} .
$$

Unfortunately this argument does not generalise easily to a larger number of solutes of general valency.

2.1.1 Nonzero Pore Charge Density. In this section, we consider the case when the electro-neutrality condition in (2.21) is relaxed. The leading-order problem is then fully nonlinear in terms of the species concentration and the electric potential. The classical approach to this problem can be found in standard texts (e.g [1]), where the potential is fixed along the pore wall at the $\zeta$-potential. We focused on the case when the membrane acts as an electrical insulator. In order to simplify the analysis, we consider the situation when advection transport is small compared to diffusive transport. radius,

We then consider the nonlinear problem, where both $z$ and $r$ are scaled on the pore

$$
\begin{aligned}
\nabla^{2} C_{i}+z_{i} \nabla \cdot\left[C_{i} \nabla \phi\right] & =0 \\
\nabla^{2} \phi & =-\frac{1}{\lambda^{2}} \sum_{i} z_{i} C_{i}=-\alpha \sum_{i} z_{i} C_{i}
\end{aligned}
$$

on the domain $0<z<l_{p} / r_{p} \simeq 3 \times 10^{3}$ and $0<r<1$, subject to zero normal derivative conditions

$$
\frac{\partial C_{i}}{\partial n}=\frac{\partial \phi}{\partial n}=0
$$

on the boundary. Note that there is no time-dependence on $C_{i}, \phi$ at this order. 
If (2.19) holds, and we consider a monovalent mixture, we can then lump the positive species $p$ and the negative species $n$ as

$$
p=\sum_{z_{i}=1} C_{i}, \quad n=\sum_{z_{i}=-1} C_{i} .
$$

If we define

$$
C_{+}=\sum_{z_{i}=1} C_{i}(0) e^{-\phi(0)}, \quad C_{-}=\sum_{z_{i}=-1} C_{i}(0) e^{\phi(0)},
$$

and with a shift in the potential by $\phi^{*}=\left[\log \left(C_{-} / C_{+}\right)\right] / 2$, we find the following nonlinear eigenvalue problem for the shifted potential $\phi$

$$
\nabla^{2} \phi=-\gamma \sinh \phi .
$$

The two simplest classes of solutions for this problem are purely axial solutions and purely radial solutions. For purely axial solutions, let us consider the simplest problem with Dirichlet boundary conditions

$$
\begin{array}{r}
\frac{d^{2} \phi}{d z^{2}}+\gamma \sinh \phi=0, \quad 0<z<\frac{l_{p}}{r_{p}}, \\
\phi(0)=\phi_{0}, \quad \lim _{z \rightarrow l_{p} / r_{p}} \phi=\phi_{1} .
\end{array}
$$

Equation (2.33) can be integrated once, and from the constant of integration, we find the relation between the field strength to the difference in potential

$$
\left.\left(\frac{d \phi}{d z}\right)^{2}\right|_{z=0}-\left.\left(\frac{d \phi}{d z}\right)^{2}\right|_{z \rightarrow l_{p} / r_{p}}=2 \gamma\left[\cosh \phi_{1}-\cosh \phi_{o}\right] .
$$

From this relation, we note that the classical results arise depending on the value of $\gamma$. In the limit $\gamma \gg 1$, then $\phi_{1}=\phi_{0}$, or the pore acts like a conductor. In the limit $\gamma \ll 1$, then $\left|\phi_{z}\right|_{z=0}=\left|\phi_{z}\right|_{z \rightarrow l_{p} / r_{p}}$, which corresponds to a continuous electric field along the membrane, or the membrane acts like a perfect dielectric.

The purely radial problem

$$
\frac{1}{r} \frac{d}{d r}\left[r \frac{d \phi}{d r}\right]=-\gamma \sinh \phi(r)
$$

needs to be solved numerically. The eigenvalue $\gamma$ is found by optimizing over the unknown reference potential $\phi(0)$.

2.2 Modified Solvent Flow. Osmosis through a semi-permeable membrane is described directly in elementary chemistry courses for nonelectrolytes. A membrane is designed to allow solvent molecules to pass through, but prohibit the transport of larger solute molecules. Due to the estimates in kinetic theory, the number of impacts per molecule on either side of the membrane is approximately the same, but the side which has a lower concentration of solute will have a larger number of solvent molecules striking the membrane. Since the solvent molecules can pass through, this results in a net flow of solvent from the low concentration side of the barrier to the higher concentration side. From [2], the accepted equation for the volume flux of solvent in such a system is given by

$$
Q_{p}=L_{p} \Delta P_{\infty}-L_{\Pi} \Delta \Pi_{\infty},
$$

where $Q_{p}$ is the volume flux of the solute, $L_{p}$ is a hydraulic coefficient, and the notation $\Delta P_{\infty}$ denotes the jump in bulk values of the hydraulic pressure $P$ across the membrane. 
Table 6 Typical osmotic pressure drops found from bulk plasma and dialysate values, assuming that there are no electrical effects. Note the bias in the hydrostatic pressure given in Table 2 .

\begin{tabular}{|l||c|c|c|}
\hline Species & Plasma (meq/L) & Dialysate $(\mathrm{meq} / \mathrm{L})$ & $\Delta \Pi(\mathrm{kPa})$ \\
\hline \hline Sodium & 140 & 140 & 0 \\
\hline Potassium & 5 & 3 & 5 \\
\hline Chloride & 114 & 110 & 10 \\
\hline Bicarbonate & 20 & 35 & -37 \\
\hline Urea & 40 & 0 & 99 \\
\hline
\end{tabular}

The additional term corresponds to the "osmotic pressure" $\Pi=R T C_{\infty}$, and $L_{\Pi}$ is a conductivity coefficient. For a perfect semi-permeable membrane, $L_{\Pi}=L_{p}$. Note that the scale for $\Pi$ is on the order of $0.35 \mathrm{MPa}$, which is about one order of magnitude larger than the characteristic hydraulic pressure scale. In the case when the membrane is "leaky", or some solute is allowed to pass through the membrane, $L_{p}>L_{\Pi}$. Note that these leaky membranes are the standard approach to simple models of the dialysis process with spatial variation (see [4]).

Note that the dialysis process is designed for the removal primarily of urea from the blood. The osmotic pressures for urea, based on its concentration, is below the hydraulic pressure difference between the blood and the dialysate (see Table 2.2). This suggests that the membranes used in dialysis are leaky, and the coefficient $L_{\Pi}$ needs to be determined based on the concentration and electric potential effects within the pore. In principle, there should be a single model that describes the flow of solvent in this situation for the mixture of charged species, and we focus on this topic in this section.

We follow [2] in theme, but consider the case of charged species to find how the concentration gradients determine the fluid velocity in the pore. We begin by considering the the momentum and continuity equations for fluid flow in the pore, assuming fluid inertial effects are negligible (see [1])

$$
\begin{aligned}
\nabla \cdot \mathbf{u} & =0, \\
-\nabla p+\mu \nabla^{2} \mathbf{u}+\rho_{E} \mathbf{E} & =0,
\end{aligned}
$$

on $0<r<r_{p}, 0<z<l_{p}$, where $\mathbf{u}=u \mathbf{r}+w \mathbf{z}$ is the velocity field in the pore, $p$ is the fluid pressure, $\mu$ is the dynamic viscosity, $\rho_{E}=\sum_{i} z_{i} e F C_{i}$ is the charge density, and $\mathbf{E}=-\nabla \phi$ is the electric field.

We scale $r$ on $r_{p}, z$ on $l_{p}, w$ on $w_{o}=\delta\left[C_{o} F k_{B} T r_{p} / \mu\right], p$ on $p_{o}=\mu w_{o} l_{p} / r_{p}^{2}$, and consider the same asymptotic series expansion as in Section 2.1. For each of the concentration fields, Nerst's relation (2.19) holds (written here in dimensional form for clarity)

$$
C_{i}=C_{o} A_{i}(z, t) e^{-z_{i} F\left(\phi(r, z, t)-\Phi^{+}\right) / R T} .
$$

Note that from this form, the osmotic pressures can be found formally as

$$
\Pi_{i}=R T C_{i}=-z_{i} F \int C_{i} d \phi,
$$

which suggests that the $A_{i}(z, t)$ are effectively the nondimensional partial osmotic pressure of species $i$ in the pore. 
From the leading order $r$-momentum equation, we have

$$
\frac{\partial p}{\partial r}+\sum_{i} z_{i} n_{o i} \frac{\partial \phi}{\partial r}=0
$$

which has a first integral that gives

$$
p(r, z, t)=P_{o}(z, t)-\sum_{i} A_{i}(z, t) e^{-z_{i} \phi(r, z, t)} .
$$

To consider the $z$-momentum equations, we note that

$$
\frac{\partial p}{\partial z}=\frac{\partial P_{o}}{\partial z}-\sum_{i}\left(\frac{\partial A_{i}}{\partial z}-z_{i} A_{i} \frac{\partial \phi}{\partial z}\right) e^{-z_{i} \phi}
$$

which results in the following problem for the axial fluid velocity $w$

$$
\frac{1}{r} \frac{\partial}{\partial r}\left(r \frac{\partial w}{\partial r}\right)=\frac{\partial P_{o}}{\partial z}-\sum_{i} \frac{\partial A_{i}}{\partial z} e^{-z_{i} \phi(r, z, t)},
$$

with $\lim _{r \rightarrow 0} r \partial w / \partial r=0$ and $w=0$ along the pore wall $r=1$. Formally, the solution can be written in terms of the following integral

$$
w=\int_{r}^{1} \frac{1}{r_{2}} \int_{0}^{r_{2}} r_{1}\left(\frac{\partial P_{o}}{\partial z}-\sum_{i} \frac{\partial A_{i}}{\partial z} e^{-z_{i} \phi\left(r_{1}, z, t\right)}\right) d r_{1} d r_{2} .
$$

To solve for $u$, we use the continuity equation (2.36) with the expression of $w$ above. Requiring that both $u=0$ along $r=0$ and $r=1$ gives a constraint for $P_{o}$ in terms of the electric potential $\phi$. However, in this general form, the transport equations for $n_{0 i}$ along with Gauss's equation for $\phi$ are highly coupled.

As a simple example, let us assume electro-neutrality in the pore, which gives $\phi=\phi(z, t)$. Note that the pressure $p=p(z, t)$ still has the osmotic terms in its expression, and the expression for $w$ in this case becomes

$$
w=\frac{r^{2}-1}{4}\left(\frac{\partial P_{o}}{\partial z}-\sum_{i} \frac{\partial A_{i}}{\partial z} e^{-z_{i} \phi(z, t)}\right) .
$$

From (2.36), we find that $u$ is given by

$$
u=-\left(\frac{r^{3}}{16}-\frac{r}{8}\right) \frac{\partial}{\partial z}\left\{\frac{\partial P_{o}}{\partial z}-\sum_{i} \frac{\partial A_{i}}{\partial z} e^{-z_{i} \phi}\right\},
$$

and the requirement that $u=0$ along $r=1$ gives the following Reynolds equation for $P_{o}$

$$
\frac{\partial}{\partial z}\left(\frac{\partial P_{o}}{\partial z}-\sum_{i} \frac{\partial A_{i}}{\partial z} e^{-z_{i} \phi}\right)=0 .
$$

One integration gives that the flow rate through the pore is constant, or

$$
Q_{p}=-\frac{1}{16}\left(\frac{\partial P_{o}}{\partial z}-\sum_{i} \frac{\partial A_{i}}{\partial z} e^{-z_{i} \phi}\right) .
$$

A second integral, if possible, would give the leaky flux relation for solvent in terms of the bulk hydraulic pressure and the bulk concentrations of each species. Note, however, that the constant flow rate then gives a velocity profile of the form (2.4), which suggests that the results of Section 2.1 hold for this particular example. 


\section{Porous-Medium Membrane Model}

In this section, the motion of the fluid through the cartridge is assumed to be pressure driven. Opposing pressure gradients are imposed at both ends of the device in order to force the blood and the dialysate to travel in opposite directions. Furthermore, the reference pressure of the blood exceeds the pressure of the dialysate, resulting in fluid from the blood flowing through the membrane and into the dialysate solution. The red blood cells are unable to penetrate the membrane and as a result, their concentration in the fibre increases. The goal of this section is to compute the large scale flow within a single fibre and the surrounding dialysate. Using this fluid velocity profile, the concentration of red blood cells in the fibre can be obtained. How the concentration of solute varies with the removal of the plasma is left for a later work.

3.1 A Simple Model. To describe the essential features of the large scale flow, we consider the two dimensional motion of fluid that is confined between two adjacent regions. These regions are separated by a permeable membrane (see Figure 3). One of these regions represents the interior of the fibre where the blood flows. The other represents the exterior region of dialysate solution. We apply symmetry conditions along $z= \pm r_{t}$. For simplicity, we assume that the "radius" of the dialysate region is equal to the radius of the fibre. Changing this value should not have a significant quantitative effect on the dynamics of the system. The length of the fibre, $l_{d}$, is much greater than the radius $r_{t}$ in the cartridge, and this fact will be used to simplify the governing equations. Since the flow is pressure driven, we further assume that the pressure is prescribed at both ends of both regions.

The fluid in both regions is assumed to be modelled by the steady, incompressible, Navier-Stokes equations. In two spatial dimensions, these can be written as

$$
\begin{gathered}
\rho(\mathbf{u} \cdot \nabla) u=-\frac{\partial p}{\partial x}+\mu \nabla^{2} u \\
\rho(\mathbf{u} \cdot \nabla) w=-\frac{\partial p}{\partial z}+\mu \nabla^{2} w \\
\frac{\partial u}{\partial x}+\frac{\partial w}{\partial z}=0
\end{gathered}
$$

where $\mathbf{u}=u(x, z) \mathbf{x}+w(x, z) \mathbf{z}$ is the fluid velocity vector written in terms of components along the standard Cartesian unit vectors, $\rho$ is the fluid density, $p$ is the hydrodynamic pressure, $\mu$ is the dynamic viscosity of the fluid, and $\nabla$ is the gradient operator in Cartesian coordinates. The velocity of the blood and of the dialysate is denoted by $\mathbf{u}_{1}$ and $\mathbf{u}_{2}$, respectively. Similarly, the pressure in the blood and in the dialysate is labelled as $p_{1}$ and $p_{2}$. For simplicity, the density of the two fluids, as well as their viscosities, are assumed to be equal. The concentration of red blood cells, $b(x, z)$, is governed by a steady advectiondiffusion equation

$$
\nabla \cdot \mathbf{J}=0, \quad \mathbf{J}=\mathbf{u}_{1} b-D \nabla b
$$

where $\mathbf{J}=\mathbf{J}(x, z)$ is the flux of red blood cells and $D$ is the diffusion coefficient. This equation only holds in the interior of the fibre, since red blood cells cannot pass through the membrane. 


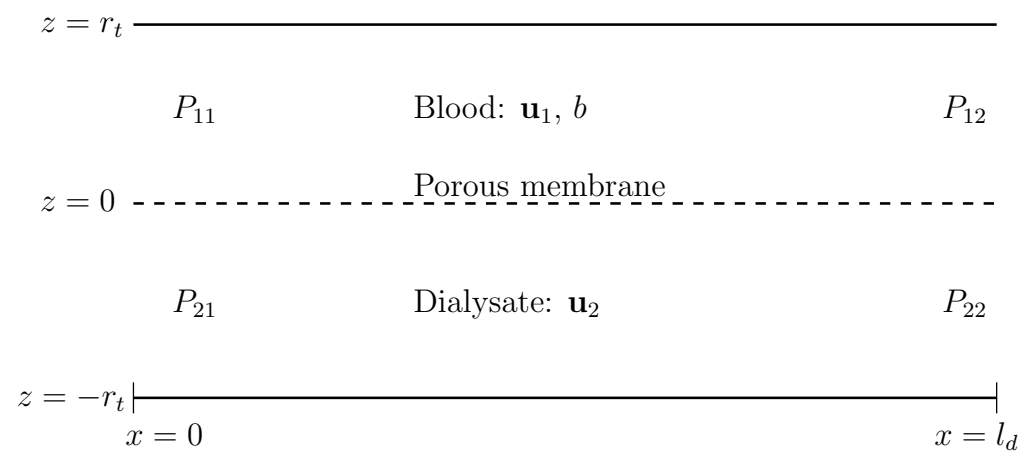

Figure 3 Model geometry. The top region corresponds to the interior of the fibre where the blood is flowing, whereas the bottom corresponds to the dialysate region exterior to the fibre. The length and the radius of the fibre are denoted by $l_{d}$ and $r_{t}$, respectively. Governing equations will model fluid velocities $\mathbf{u}_{1}$ and $\mathbf{u}_{2}$ in the blood and dialysate regions, respectively, as well as the blood cell concentration $b$ inside the fibre. The $P_{i j}$ denote prescribed fluid pressures at the ends of the system.

The symmetry of the system implies that there is a stress-free condition and a no-flow condition on the fluid velocities at the center of the regions,

$$
\begin{aligned}
& \frac{\partial u_{1}}{\partial z}=w_{1}=0, \quad z=r_{t} \\
& \frac{\partial u_{2}}{\partial z}=w_{2}=0, \quad z=-r_{t} .
\end{aligned}
$$

Along the membrane, there is a no-slip condition on the horizontal fluid velocities , $u_{1}=$ $u_{2}=0$ along $z=0$. Since fluid can pass through this membrane, the vertical velocity is governed by Darcy's law at $z=0$, which states that the vertical fluid velocity at the membrane is proportional to the pressure difference across it

$$
w_{1}=w_{2}=\frac{k}{\mu} \frac{p_{2}-p_{1}}{l_{p}}, \quad z=0,
$$

where $k$ and $l_{p}$ are the permeability and the thickness of the membrane, respectively. The permeability is assumed to be constant, which implies that none of the pores in the membrane become blocked by red blood cells. It is further assumed that the pressure at the ends of each region is prescribed,

$$
\begin{aligned}
& p_{i}=P_{i 1}, \quad x=0, \\
& p_{i}=P_{i 2}, \quad x=l_{d},
\end{aligned}
$$

for $i=1,2$. To obtain the correct flow, the following inequalities are assumed to be true

$$
P_{21}<P_{22}<P_{12}<P_{11} \text {. }
$$

These are needed to ensure that the blood and the dialysate flow in opposite directions and they allow the water from the blood to cross the membrane.

It is assumed that the concentration of red blood cells entering the device is a fixed constant $b_{0}$. Furthermore, there is zero flux of red blood cells at $z=0$ and $z=r_{t}$. The condition at $z=0$ arises because red blood cells cannot pass through the membrane and the condition at $z=l$ is from the symmetry. In summary, the boundary conditions for the 
concentration are given by

$$
\begin{gathered}
b=b_{0}, \quad x=0, \\
\mathbf{J} \cdot \mathbf{n}=0, \quad z=0, r_{t},
\end{gathered}
$$

where $\mathbf{n}$ is a unit vector normal to the boundary. Although the equation which governs the concentration (3.2) is second order in $x$, further analysis will show that only one boundary condition is required.

3.2 Scaling and Nondimensionalization. One of the characteristic properties of each fibre is its length. Typically, a fibre is approximately one hundred times longer than it is wide. Therefore, we define the small parameter $\varepsilon=r_{t} / l_{d} \ll 1$ and rescale the governing equations. In particular, we scale the physical dimensions by $x \rightarrow l_{d} x, z \rightarrow r_{t} z$, where the new, nondimensional, domain is from $0 \leq x \leq 1,-1 \leq z \leq 1$. The velocity components and the pressure are scaled according to

$$
u \rightarrow U_{b} u, \quad w \rightarrow \varepsilon U_{b} w, \quad p \rightarrow \frac{\mu U_{b}}{r_{t} \varepsilon} p,
$$

where $U_{b}$ is the mean velocity of the blood through the device. With these new variables, the Navier-Stokes equations (3.1b) become

$$
\begin{aligned}
\varepsilon^{2} \operatorname{Re}(\mathbf{u} \cdot \nabla) u= & -\frac{\partial p}{\partial x}+\varepsilon^{2} \frac{\partial^{2} u}{\partial x^{2}}+\frac{\partial u}{\partial z} \\
\varepsilon^{4} \operatorname{Re}(\mathbf{u} \cdot \nabla) w= & -\frac{\partial p}{\partial z}+\varepsilon^{2}\left(\varepsilon^{2} \frac{\partial^{2} w}{\partial x^{2}}+\frac{\partial^{2} w}{\partial z^{2}}\right), \\
& \frac{\partial u}{\partial x}+\frac{\partial w}{\partial z}=0,
\end{aligned}
$$

where the Reynolds number is defined as

$$
\operatorname{Re}=\frac{\rho U_{b} l_{d}}{\mu} .
$$

From Table 1, Re $\sim O(1)$. Therefore, to leading order, the pressure along the $z$ direction is constant, and the pressure gradient in the $x$ direction is balanced by viscous diffusion in the $z$ direction. The boundary conditions essentially remain unchanged, except for scaling the constant values in (3.4) and scaling Darcy's law in (3.3) to become

$$
w=K\left(p_{2}-p_{1}\right),
$$

where $K=k / r_{t} l_{p} \varepsilon^{2} \sim O(1)$.

The concentration can be rescaled according to $b \rightarrow b_{0} b$, resulting in the nondimensional advection-diffusion equation

$$
\varepsilon^{2} \operatorname{Pe}\left(\mathbf{u}_{1} \cdot \nabla\right) b=\varepsilon^{2} \frac{\partial^{2} b}{\partial x^{2}}+\frac{\partial^{2} b}{\partial z^{2}}
$$

where the Péclet number for the blood cells is given by

$$
\mathrm{Pe}_{\mathrm{b}}=\frac{U_{b} l_{d}}{D} \sim O\left(\varepsilon^{-2}\right) .
$$

Thus, to leading order, the advection of red blood cells is balanced by their diffusion in the $z$ direction. The boundary conditions simplify to become $b=1$ at $x=0$ and

$$
\varepsilon^{2} \operatorname{Pe} w b-b_{z}=0, \quad z=0,1 .
$$


As can be seen from (3.8), the second order derivative in $x$ drops out. Indeed, only a single boundary condition in $x$ is needed to obtain a solution at leading order.

3.3 Solution. To leading order, (3.6) suggests that $p=p(x)$. In addition, (3.5) gives the following velocity profiles in the axial direction in each region

$$
\begin{aligned}
& u_{1}=\frac{d p_{1}}{d x}\left(\frac{z^{2}}{2}-z\right), \\
& u_{2}=\frac{d p_{2}}{\partial x}\left(\frac{z^{2}}{2}+z\right) .
\end{aligned}
$$

Conservation of mass (3.7), along with the no-flow conditions at $z= \pm 1$ gives the vertical velocity component in each domain

$$
\begin{aligned}
& w_{1}=\frac{d^{2} p_{1}}{d x^{2}}\left(\frac{-z^{3}}{6}+\frac{z^{2}}{2}-\frac{1}{3}\right), \\
& w_{2}=\frac{d^{2} p_{2}}{d x^{2}}\left(\frac{-z^{3}}{6}-\frac{z^{2}}{2}+\frac{1}{3}\right) .
\end{aligned}
$$

Applying the condition on $w$ at $z=0$ shows the pressure must satisfy the system of ordinary differential equations given by

$$
\begin{aligned}
& \frac{d^{2} p_{1}}{d x^{2}}=3 K\left(p_{1}-p_{2}\right), \\
& \frac{d^{2} p_{2}}{d x^{2}}=3 K\left(p_{2}-p_{1}\right) .
\end{aligned}
$$

Each solution takes the form $p_{i}(x)=a_{i, 1} e^{\sqrt{6 K} x}+a_{i, 2} e^{-\sqrt{6 K} x}+a_{i, 3} x+a_{i, 4}$. The coefficients are cumbersome functions of both $K$ and the pressure's boundary conditions, and showing them here would provide no further insight.

Because the flow is assumed to be two dimensional and incompressible, stream functions $\psi_{i}$ can be found. Solving $u=\psi_{z}$ and $w=-\psi_{x}$ in each region yields the two stream functions

$$
\begin{aligned}
& \psi_{1}=\frac{d p_{1}}{d x}\left(\frac{z^{3}}{6}-\frac{z^{2}}{2}+\frac{1}{3}\right), \\
& \psi_{2}=\frac{d p_{2}}{d x}\left(\frac{z^{3}}{6}+\frac{z^{2}}{2}-\frac{1}{3}\right) .
\end{aligned}
$$

Using the fluid velocity found above, the concentration of red blood cells in the upper region can be solved. Despite the governing equation being linear, the velocities are sufficiently complicated that it could not be solved analytically. Instead, a simple, upwinded, finite differencing scheme was used. In particular, central differencing was used in the $z$ direction and backwards differencing was used in the $x$ direction to account for the blood travelling in the positive $x$ direction.

3.4 Results and Discussion. The scaled streamlines can be seen in Figure 4. Blood enters the top region from the left and has a small downward component, which is a result of the prescribed pressure differences on the left and right boundaries and the porous membrane. In the bottom region, dialysate enters from the right and also has a small downward component. The flow from the top to the bottom region is expected, as this corresponds 


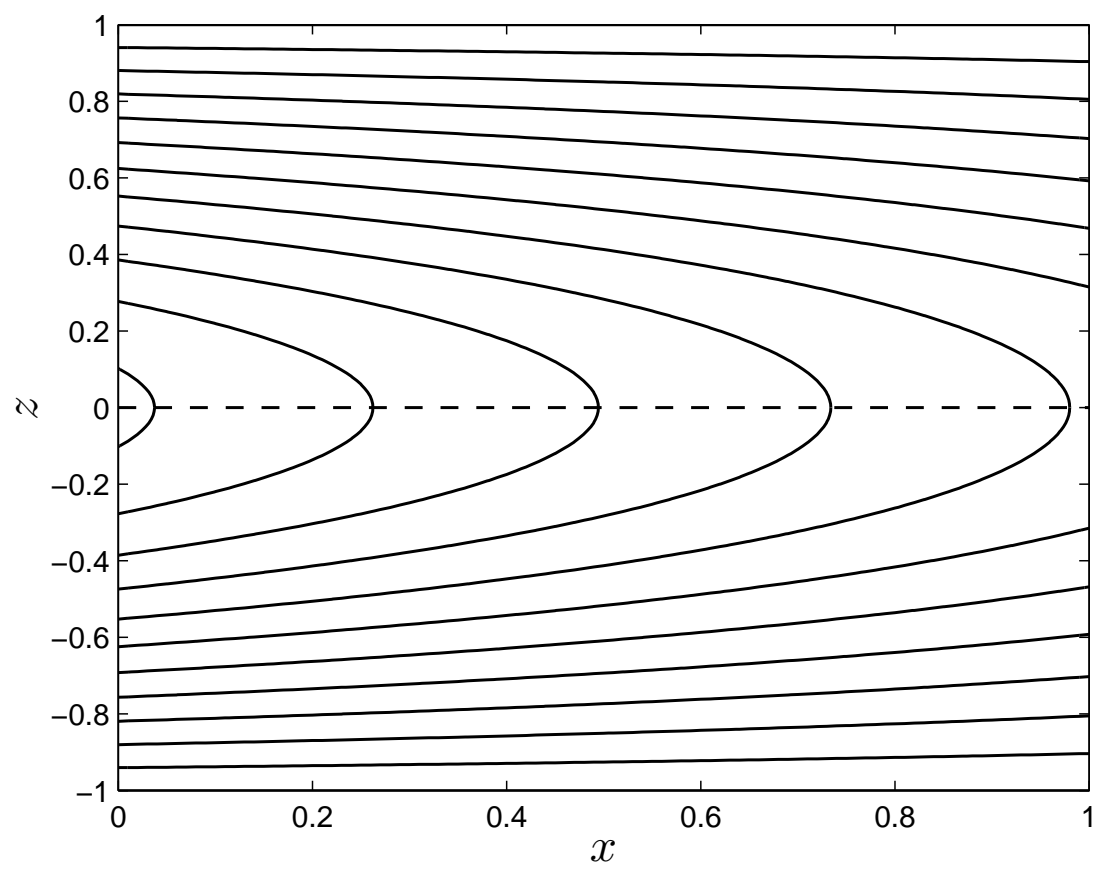

Figure 4 Scaled streamlines of the flow in two regions separated by a porous membrane (dashed line).

to plasma water leaving the inside of the hollow fibres and entering the dialysate fluid space, which is what happens during the dialysis process. This flow represents the underlying convectively dominated régime of solute transport, assuming that electrical effects are negligible.

The pressure found depends only on $x$, and although it is an exponential function, the value of $K$ is small enough for the function to be approximately linear in both regions. If $K$ is increased enough, there is a pressure drop in the center, and some of the fluid from the right of the top region will flow to the left and then down into the bottom. Since $K$ is proportional to permeability, this intuitively means that increasing the permeability of the membrane makes it harder for the fluid in the top region to make it across. Furthermore, as the permeability tends to zero, the pressure becomes a linear function of $x$, and both velocity profiles reduce to Poiseuille flow in Cartesian coordinates.

In Figure 5, the concentration gradient of red blood cells in the upper region can be seen, along with the stream lines. The concentration increases toward the membrane and also increases downstream to the right. Interestingly, the concentration gradient appears everywhere to be almost parallel to the streamlines. This implies a large contribution to the movement of red blood cells from diffusion, otherwise we would expect to see concentrations that are constant along the streamlines. In fact, this solution is valid not only for red blood cells, but for any solute that cannot pass through the membrane, such as protein. This is provided the diffusion coefficient of the solute is on the same order as that for red blood cells and, if the solute is charged, electric effects can be ignored. 


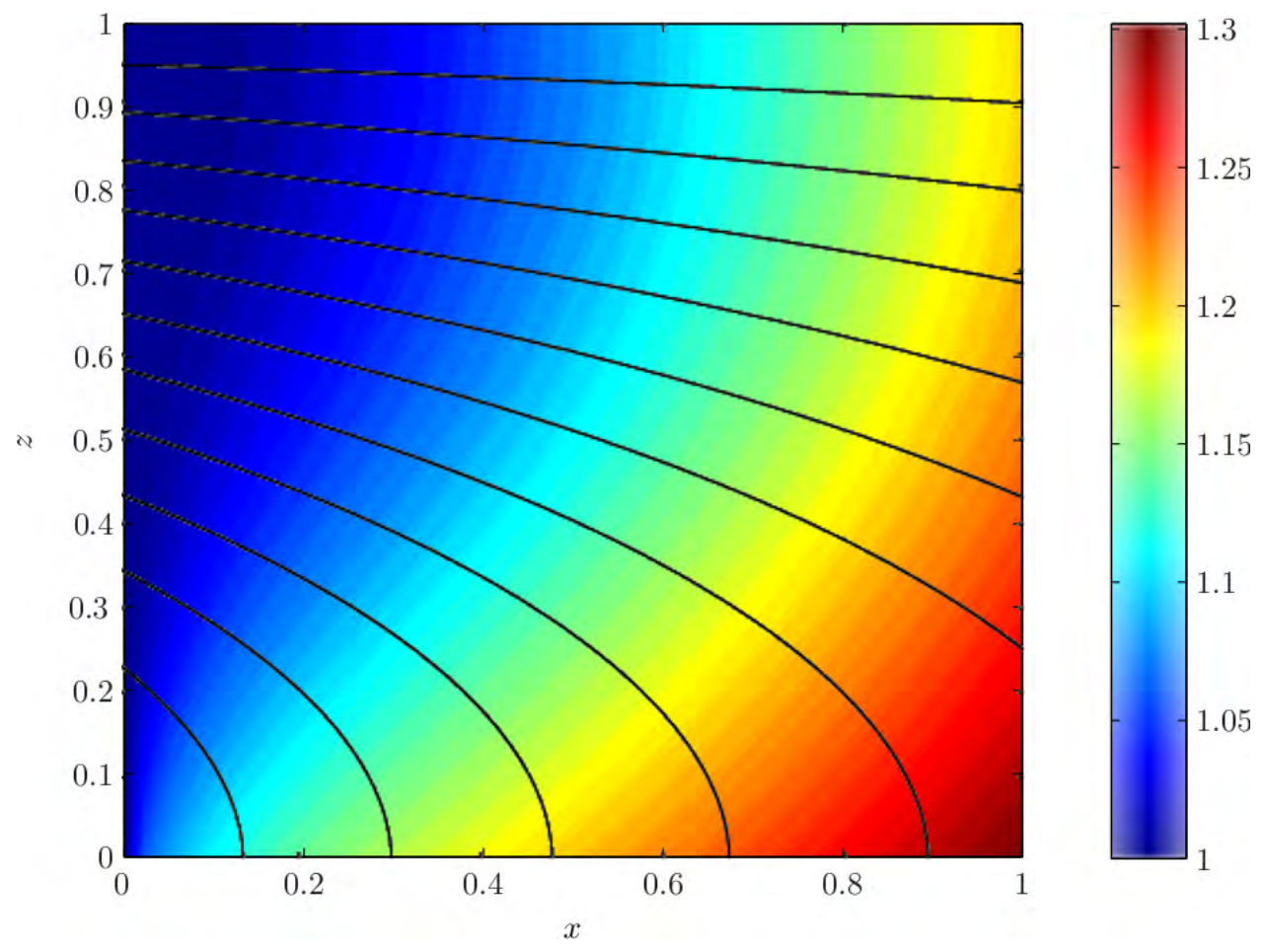

Figure 5 Scaled blood-cell concentration gradient and the streamlines in the fibre.

\section{Bulk Solute Transport - Outer Solutions}

In this section, we review the different models that try to estimate the solute distribution away from the membrane. In all of these models, since the advective transport to the membrane is much smaller than the advective transport along the filament axis, the effective equations of motion are quasi-steady. In future work, additional details on how advection of solute from the blood into the dialysate would need to be included in order to see changes on this longer time scale.

\subsection{Toy model based on transport equilibrium.}

4.1.1 Assumptions. Our simplest model of a dialyzer assumes that:

1. The transport of solutes between the blood and the dialysate involves the sum of a diffusive term (proportional to the concentration difference across the tube walls), and an electrical term (proportional to the product of the charge on the species, the electric field across the tube walls, and the average concentration).

2. Dialysis continues for sufficient time to allow the blood to come in to equilibrium with the dialysate.

3. Electro-neutrality is maintained in the blood and dialysate at leading order (though minute differences can lead to a significant potential difference, and hence an electric field, across the tube walls).

4.1.2 Notation and equations. Consider $n$ diffusing species. Each species $i \in\{1,2, \ldots n\}$ has valencey $z_{i}$, and concentrations $C_{i}$ in the blood and $c_{i}$ in dialysate initially. Since the 
Table 7 Initial concentrations $C_{i}$ in the blood and $c_{i}$ in the dialysate, used in the example in Section 4.1.3.

\begin{tabular}{|c||c|c|c|c|}
\hline$i$ & Species & $z_{i}$ & $C_{i}(0)$ & $c_{i}$ \\
\hline \hline 1 & $\mathrm{Na}^{+}$ & +1 & 100 & $c$ \\
\hline 2 & $\mathrm{~K}^{+}$ & +1 & 1 & 0 \\
\hline 3 & $\mathrm{Cl}^{-}$ & -1 & 100 & $c$ \\
\hline 4 & $\mathrm{Pr}^{-}$ & -1 & 1 & 0 \\
\hline
\end{tabular}

dialysate is not recycled, the concentration there remains fixed at $c_{i}$, while the concentration in the blood can evolve to its equilibrium value $C_{i}^{\infty}=\lim _{t \rightarrow \infty} C_{i}(t)$.

The equations for our model, to determine the equilibrium concentrations $C_{i}^{\infty}$ are then as follows. For species that can pass through the tube walls we must have no net transport at equilibrium. We assume that the advective, diffusive and electrical fluxes from the blood to the dialysate are given, respectively, by

$$
\begin{aligned}
Q_{\mathrm{a}} & =Q\left(\frac{C_{i}+c_{i}}{2}\right), \\
Q_{\mathrm{d}} & =D_{i}\left(C_{i}-c_{i}\right), \\
Q_{\mathrm{e}} & =\frac{q z_{i} D_{i}}{k_{B} T}\left(\frac{C_{i}+c_{i}}{2}\right) E,
\end{aligned}
$$

where $Q$ is the flux of fluid through the wall, $E$ is the mean electric field strength, $D_{i}$ is a species dependent mobility coefficient for passing through the tube walls. At equilibrium, the total flux of each species must be zero. We also have no flux of fluid, so $Q=0$ and there is no advective transport to consider. Balancing the diffusive and electrical fluxes we find that

$$
D_{i}\left[\left(C_{i}^{\infty}-c_{i}\right)+\frac{q z_{i}}{k_{B} T}\left(\frac{C_{i}^{\infty}+c_{i}}{2}\right) E\right]=0 .
$$

For species that are unable to pass through the tube walls, the concentration in the blood cannot alter, so

$$
C_{i}^{\infty}=C_{i} .
$$

Finally the condition of electro-neutrality gives us

$$
\sum_{i} C_{i}^{\infty} z_{i}=0
$$

We now have $n+1$ equations for the $n+1$ unknowns $\left\{C_{i}^{\infty}\right\}$ and $E$.

4.1.3 A simple example. For simplicity we consider just four diffusing species: $\mathrm{Na}^{+}, \mathrm{K}^{+}$, $\mathrm{Cl}^{-}$, and $\mathrm{Pr}^{-}$(the latter representing a negatively charged protein ion). All the ions are monovalent $\left(z_{i}= \pm 1\right)$, and the first three can pass through the tube walls, but the protein cannot. Initial concentrations in the blood and dialysate are shown in Table 4.1.3. The blood contains a mixture of all four, whereas the dialysate contains only a concentration $c$ of $\mathrm{NaCl}$.

We want to find appropriate concentration $c$ of $\mathrm{NaCl}$ in dialysate to allow removal of $\mathrm{K}^{+}$without affecting $\mathrm{Na}^{+}$levels. But firstly we solve for the forward problem to determine $\left\{C_{i}^{\infty}\right\}$ for a given $c$. 


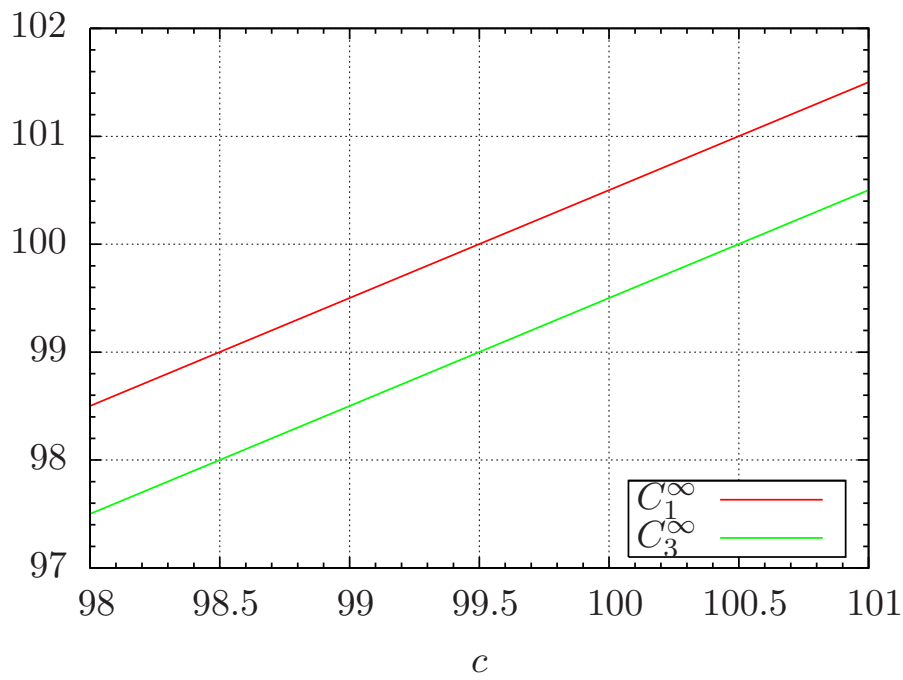

Figure 6 The equilibrium concentrations $\mathrm{Na}^{+}\left(C_{1}^{\infty}\right)$ and $\mathrm{Cl}^{-}\left(C_{3}^{\infty}\right)$ in the blood as functions of $\mathrm{NaCl}$ concentration $c$ in the dialysate for the simple example in Section 4.1.3.

The equations are as follows:

$$
\begin{aligned}
\left(C_{1}^{\infty}-c\right)+E^{\prime}\left(C_{1}^{\infty}+c\right) & =0, \\
C_{2}^{\infty}\left(1+E^{\prime}\right) & =0, \\
\left(C_{3}^{\infty}-c\right)-E^{\prime}\left(C_{3}^{\infty}+c\right) & =0, \\
C_{4}^{\infty}-1 & =0, \\
C_{1}^{\infty}+C_{2}^{\infty}-C_{3}^{\infty}-C_{4}^{\infty} & =0,
\end{aligned}
$$

where $E^{\prime}=q E / k_{B} T$.

From (4.8) either $E^{\prime}=-1$ or $C_{2}^{\infty}=0$. The former is ruled out by (4.7), since this equation could then only be satisfied if $c=0$. Hence $C_{2}^{\infty}=0$. We can then eliminate $E^{\prime}$ between (4.7) and (4.9) to obtain a pair of equations for $C_{1}^{\infty}$ and $C_{3}^{\infty}$ :

$$
\begin{aligned}
\frac{C_{1}^{\infty}-c}{C_{1}^{\infty}+c} & =-\frac{C_{3}^{\infty}-c}{C_{3}^{\infty}+c}, \\
C_{1}^{\infty} & =1+C_{3}^{\infty} .
\end{aligned}
$$

The solution for $C_{1}^{\infty}$ and $C_{3}^{\infty}$ in terms of $c$ is now straightforward:

$$
\begin{aligned}
C_{1}^{\infty} & =\frac{\sqrt{1+4 c^{2}}+1}{2}, \\
C_{3}^{\infty} & =\frac{\sqrt{1+4 c^{2}}-1}{2} .
\end{aligned}
$$

These results are plotted as functions of $c$ in Figure 6.

However, we are more interested in choosing a dialysate concentration $c$ in order to obtain optimal values for $C_{1}^{\infty}$ and $C_{3}^{\infty}$. If we choose values consistent with (4.13) - necessary to ensure electro-neutrality - the value of $c$ can be computed by solving (4.12). This yields:

$$
c=\sqrt{C_{1}^{\infty} C_{3}^{\infty}} .
$$


So if we wish to obtain $C_{1}^{\infty}=100$ (so $\mathrm{Na}^{+}$levels are unaffected) and $C_{3}^{\infty}=99$ (to maintain electro-neutrality after the loss of $\mathrm{K}^{+}$), we should set the dialysate concentration of $\mathrm{NaCl}$ to be

$$
c=30 \sqrt{11} \approx 99.50 .
$$

4.2 Argument for using a 1D model. Consider a small length $\delta x$ of tube that has a concentration drop $\Delta C_{1}$ from the centre to the boundary. The average radial concentration gradient is $\Delta C_{1} / r_{t}$. If we assume advection transport of solute is small compared to diffusion, then the mass flux of solute due to diffusion at the boundary approximately equals

$$
D \frac{\Delta C_{1}}{r_{t}}\left(2 \pi r_{t} \delta x\right) \Rightarrow Q=D \frac{\Delta C_{1}}{r_{t}}\left(2 \pi r_{t}\right),
$$

where $Q$ is the mass flux per unit length of tube. The number of pores in that section of boundary is $\phi_{p}\left(2 \pi r_{t}\right) \delta x / A_{p}$, and therefore the number of pores per unit length equals $\phi_{p}\left(2 \pi r_{t}\right) / A_{p}$, meaning that we may obtain a second equation for $Q$

$$
Q=D \frac{\Delta C_{2}}{l_{p}} A_{p} \frac{\phi_{p}\left(2 \pi r_{t}\right)}{A_{p}}=D \frac{\Delta C_{2}}{l_{p}} \phi_{p}\left(2 \pi r_{t}\right),
$$

where $\Delta C_{2}$ is the concentration drop along a pore. Relating the two expressions for $Q$,

$$
\frac{\Delta C_{1}}{\Delta C_{2}}=\frac{\phi_{p} r_{t}}{l_{p}} \approx 0.02,
$$

which would suggest that most of the concentration drop takes place across the membrane. Therefore we propose to use a 1D model when transport from advection is small compared to diffusive transport.

4.3 One-dimensional steady model. The above assumption motivates a model in one dimension, in which the concentration profile of a single charged species varies in the axial direction along the tubes and along the dialysate, but not significantly in the radial direction. We consider the model depicted in Figure 7. We use a one-dimensional model and assume the flow and the concentration profiles are steady. We also make the simplifying assumption that the velocities of blood and dialysate, $U_{b}$ and $U_{d}$, respectively, are independent of $x$. This is reasonable since the filtration rate is small compared to the fluxes of blood and dialysate $\left(Q_{p} / Q_{b} \approx 1 / 24\right.$ and $\left.Q_{p} / Q_{d}=1 / 48\right)$. Table 2 suggests that the pressure drop, and hence the velocity of the blood in the pores, varies by around $20 \%$ along the length, but for simplicity we assume here that the average velocities are constant along the tubes.

In the blood we analyse a control volume consisting of a section of one tube of length $\delta x$, as shown in Figure 7. We balance the solute flux across each of the surfaces. The flux along the tube into the control volume is $A_{t i} U_{b} C_{b}(x)$, where $A_{t i}$ is the internal area of the tube and $C_{b}$ is the species concentration. The flux out of the control volume along the tube equals $A_{t i} U_{b} C_{b}(x+\delta x)$. To find the flux across the curved surface of the control volume, we find the number of pores, which equals the surface area, $2 \pi r_{t} \delta x$, multiplied by the number of pores per unit area, $n_{\text {ppua }}=N_{p} / A_{s}$, and then multiply by the flux per pore. The dimensionless flux is given by the expression $Q_{\sigma}$ appearing in (2.29), and the corresponding dimensional flux is given by $A_{p} U_{p} Q_{\sigma} / P e_{p}$. Since we are interested in the concentration of a single charged species under electrically neutral conditions, the quantities $\sigma_{b}$ and $\sigma_{d}$ appearing in (2.29) are directly proportional to $C_{b}$ and $C_{d}$ in this section. Thus the flux through 


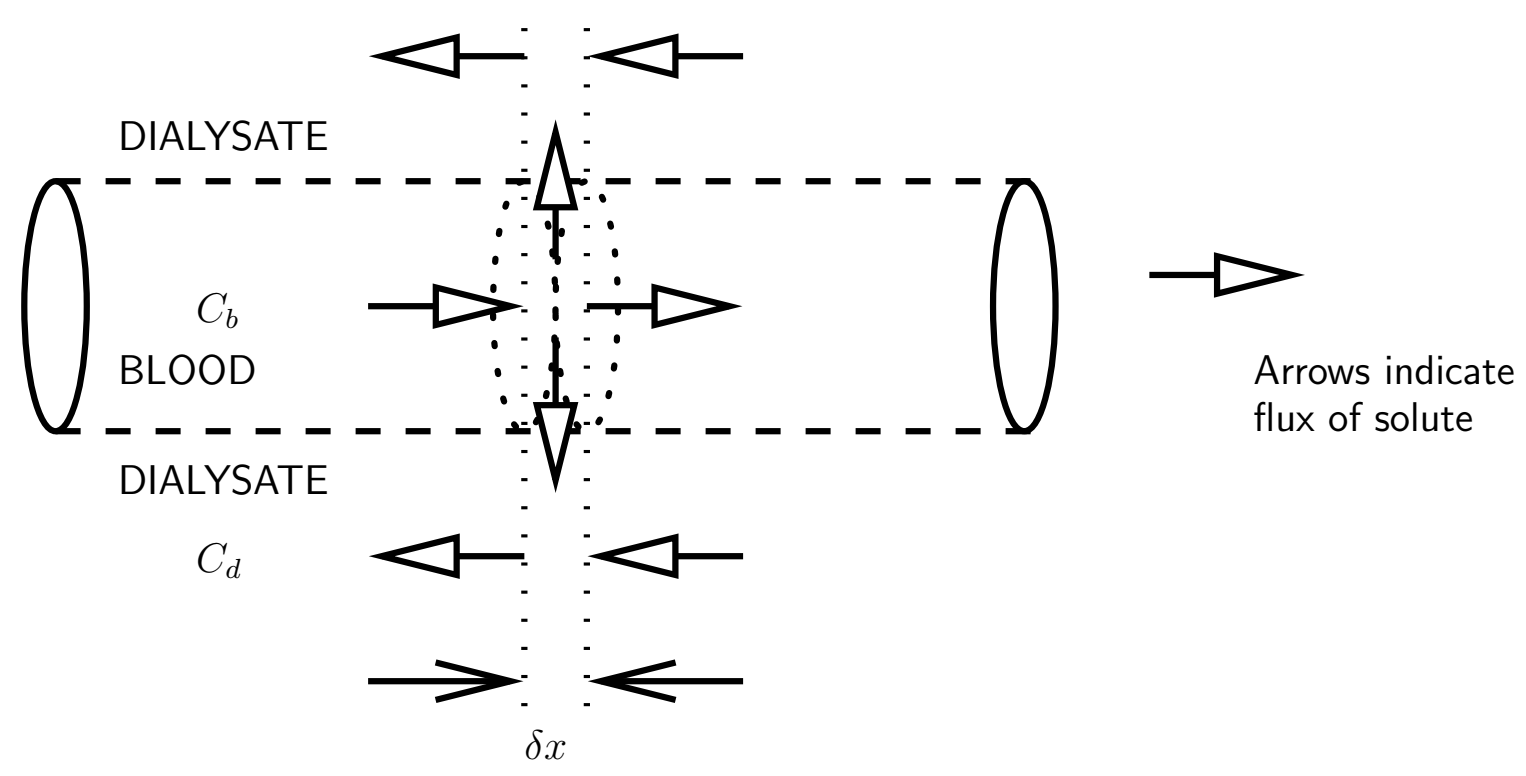

Figure 7 Schematic view of one-dimensional model, showing one tube of the dialyzer with porous walls. It is filled with blood and surrounded by dialysate.

the curved surface of the control volume equals $2 \pi r_{t} N_{p} A_{p} U_{p} Q_{\sigma} \delta x /\left(P e_{p} A_{s}\right)$. Balancing the fluxes into and out of the control volume and taking the limit as $\delta x \rightarrow 0$, we obtain

$$
\frac{\partial C_{b}}{\partial x}=-\frac{2 \pi r_{t} N_{p} A_{p} U_{p} Q_{\sigma}}{P e_{p} A_{s} A_{t i} U_{b}} .
$$

Unlike the blood, which is partitioned by the tubes, the region occupied by the dialysate is connected, so to analyse the concentration in the dialysate, we consider a control volume containing all of the dialysate between $x$ and $x+\delta x$. This is a cylindrical region with $N_{t}$ cylindrical holes in it. The flux into the control volume through the surface at $x$ is $\left(1-\phi_{t}\right) A_{d} U_{d} C_{b}(x)$, the flux through $x+\delta x$ equals $\left(1-\phi_{t}\right) A_{d} U_{d} C_{b}(x+\delta x)$, and the flux from the tubes equals $2 \pi r_{t} N_{t} N_{p} A_{p} U_{p} Q_{\sigma} \delta x /\left(P e_{p} A_{s}\right)$, meaning we obtain:

$$
\frac{\partial C_{d}}{\partial x}=-\frac{2 \pi r_{t} N_{t} N_{p} A_{p} U_{p} Q_{\sigma}}{P e_{p} A_{s}\left(1-\phi_{t}\right) A_{d} U_{d}} .
$$

Substituting the expression for $Q_{b}$ from (2.29), (4.21) and (4.22) become

$$
\begin{aligned}
\frac{\partial C_{b}}{\partial x} & =-\frac{\lambda}{\left(e^{P e_{p}}-1\right)}\left(e^{P e_{p}} C_{b}-C_{d}\right), \\
\frac{\partial C_{d}}{\partial x} & =-\frac{\lambda \kappa}{\left(e^{P e_{p}}-1\right)}\left(e^{P e_{p}} C_{b}-C_{d}\right),
\end{aligned}
$$

where

$$
\begin{gathered}
\lambda=\frac{2 \pi r_{t} N_{p} A_{p} U_{p}}{A_{s} A_{t i} U_{b}} \approx 0.043 \mathrm{~m}^{-1}, \quad \kappa=\frac{N_{t} A_{t i} U_{b}}{\left(1-\phi_{t}\right) A_{d} U_{d}} \approx 0.50, \\
P e_{p} \approx 1.8, \quad l_{d} \approx 0.2 \mathrm{~m} .
\end{gathered}
$$



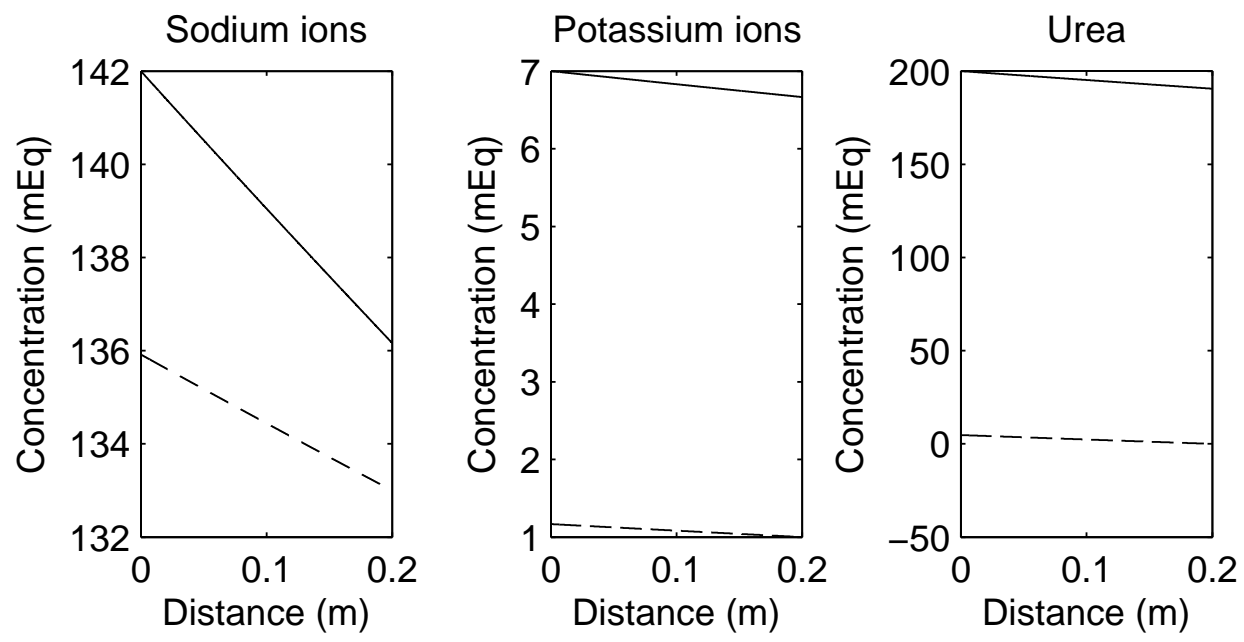

Figure 8 Concentration profiles in the blood (solid) and in the dialysate (dashed).

Table 8 Typical removal rates predicted by the model.

\begin{tabular}{|l||c|c|c|c|c|c|}
\hline Solute & $\begin{array}{c}\text { Normal } \\
\text { conc. } \\
(\mathrm{mEq})[15]\end{array}$ & $\begin{array}{c}\text { Pre- } \\
\text { dialysis } \\
\text { conc. } \\
(\mathrm{mEq})[15]\end{array}$ & $\begin{array}{c}\text { Dialysate } \\
\text { conc. } \\
(\mathrm{mEq})[15]\end{array}$ & $\begin{array}{c}\text { Diffusion } \\
\text { coefficient } \\
\left(\mathrm{m}^{2} / \mathrm{s}\right)\end{array}$ & $\begin{array}{c}\text { Removal } \\
\text { rate } \\
(\mathrm{mol} / \mathrm{s})\end{array}$ & $\begin{array}{c}\text { Mass } \\
\text { removed } \\
\text { (normal- } \\
\text { ised })\end{array}$ \\
\hline \hline Sodium & 142 & 142 & 133 & $2 \times 10^{-9}$ & $1.1 \times 10^{-25}$ & 0.78 \\
\hline Potassium & 5 & 7 & 1 & $2 \times 10^{-9}$ & $6.2 \times 10^{-27}$ & 0.04 \\
\hline Urea & $21 \mathrm{mg} / \mathrm{ml}$ & 200 & 0 & $1.8 \times 10^{-9}$ & $1.4 \times 10^{-25}$ & 1 \\
\hline
\end{tabular}

These have solution

$$
\begin{aligned}
C_{b} & =\frac{\left(e^{P e_{p}} C_{b 0}-C_{d 0}\right) e^{-\gamma x}-\kappa C_{b 0} e^{-\gamma l_{d}}+C_{d 0}}{e^{P e_{p}}-\kappa e^{-\gamma l_{d}}} \\
C_{d} & =\frac{\kappa\left(e^{P e_{p}} C_{b 0}-C_{d 0}\right) e^{-\gamma x}+e^{P e_{p}} C_{d 0}-\kappa C_{b 0} e^{P e_{p}-\gamma l_{d}}}{e^{P e_{p}}-\kappa e^{-\gamma l_{d}}},
\end{aligned}
$$

where

$$
\gamma=\frac{\lambda\left(e^{P e_{p}}-\kappa\right)}{\left(e^{P e_{p}}-1\right)}
$$

This is plotted in Figure 8 for sodium, potassium and urea, assuming that each species appears individually in solution. As can be seen, the concentration profiles show a near linear variation over the lengthscale of the dialyzer.

The total flux of the solute out of the blood is

$$
Q_{s}=\int_{0}^{l_{d}} \frac{A_{p} U_{p} Q_{\sigma}}{P e_{p}} d x=\frac{A_{p} U_{p}\left[1-e^{-\gamma l_{d}}\right]\left(e^{P e_{p}} C_{b 0}-C_{d 0}\right)}{\lambda\left(e^{P e_{p}}-1\right)\left[e^{P e_{p}}-\kappa e^{-\gamma l_{d}}\right]} .
$$

Removal rates are listed in Table 4.3

We may also calculate the ratio of advective to diffusive effects on both sides of the membrane, which is shown in Figure 9. This shows that advection is much more important than diffusion, particularly for sodium. 

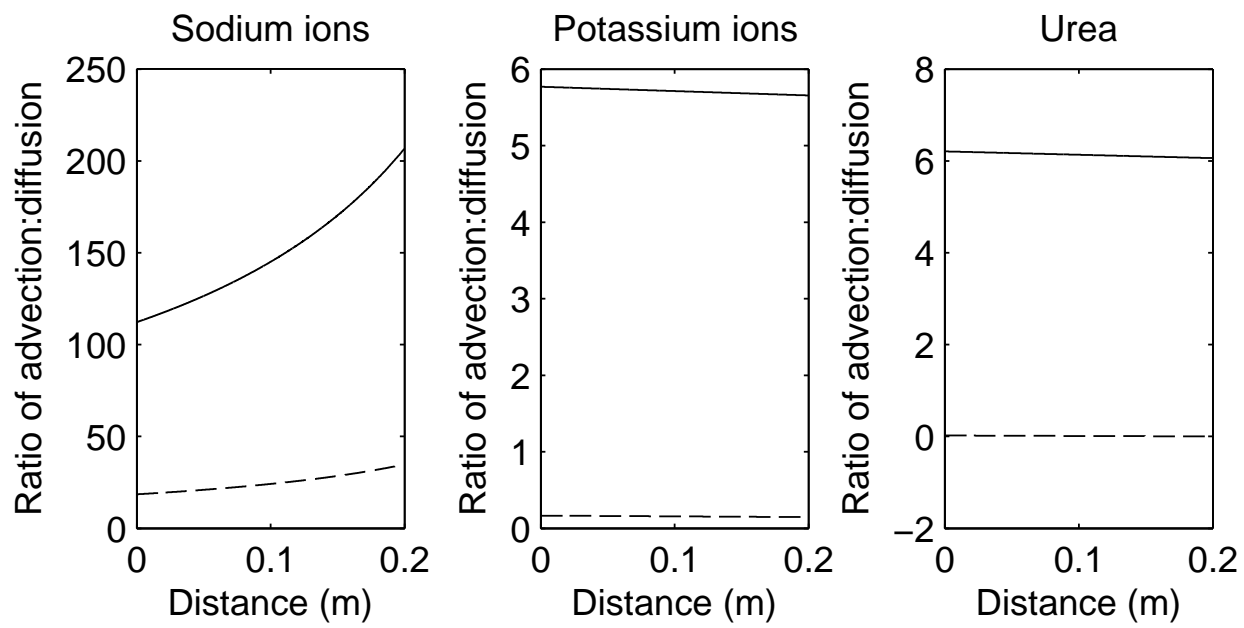

Figure 9 Ratio of advective to diffusive transport at either end (solid - blood, dashed dialysate) of the pore for the concentration profiles displayed in Figure 8, see Section 2 for details of the calculation.

\section{Conclusions}

We have investigated the transport of charged species over a variety of length scales. Firstly, we considered the transport of species through a single pore in the membrane that separates the blood from the dialysate. In the case when electro-neutrality holds, the volumetric flow rate remains constant, but depends in general on the local hydraulic and osmotic pressures and on the local electric potential. The electric potential, a streaming potential, depends only on the local solution conductivity if the Péclet numbers are identical for all species. Formally, the classic membrane relations discussed in [2] are reproduced in our analysis in this limit, but the coefficients depend nonlinearly on the solute concentration and electric potenial. In the case when electro-neutrality is weakened, there are two approaches in modeling. The first, and classical, approach is to model the charge in the membrane as a $\zeta$-potential, and prescribe this potential as a Dirichlet boundary condition along the pore wall. Our approach here considers the case of the membrane acting as a pure dielectric. In this case, we find a nonlinear eigenvalue problem for the potential, where the eigenvalue is the ratio of the pore radius to the Debye length scale.

Secondly, we considered the flow of plasma from the blood to the dialysate assuming that concentration effects were negligible. We found that the pressure difference across the dialysate decays exponentially over space, with the decay rate depending on the permeability of the membrane. Although limited to looking at the plasma effects, this model forms a foundation for developing the net charge transport across the membrane, since the solute available to cross into the dialysate is found in the plasma. Further, extensions to this model that include solute transport from the blood cells to the plasma could potentially be implemented with a local analysis of the osmotic transport across the cell wall. Although work for the future, one potential result from such a local analysis would be an effective diffusion coefficient which depends on the local blood cell concentration.

Finally, we investigated the case where the solute distribution was independent of the radial dimension. This case is appropriate if diffusive transport across the membrane is dominant over advective transport. We find that in this case, advective transport appears 
to be dominant only for the case of sodium. Since advective transport is dominant for sodium, the concentration depends not only on the axial coordinate but also on the radial coordinate, suggesting that a boundary-layer approach is needed.

\section{References}

[1] R.F. Probstein, Physicochemical Hydrodynamics: An Introduction, $2^{\text {nd }}$ Edition, Wiley Interscience, 2003.

[2] J.L. Anderson and D.M. Malone, Mechanism of osmotic flow in porous membranes, Biophys. J. 14 (1974), 957-982.

[3] J. Waniewski, Mathematical modelling of fluid and solute transport in hemodialysis and peritoneal dialysis, J. Memb. Sci. 274 (2006), 24-37.

[4] W. Ding, L. He, G. Zhao, H. Zhang, Z. Shu and D. Gao, Double porous media model for mass transfer for hemodialyzers, Int. J. Heat Mass Trans. 47 (2004), 4849-4855.

[5] S. Eloot, D. de Wachter, I van Tricht and P. Verdonck, Computational flow modeling in hollow-fiber dialyzers, Artif. Organs 26 (2002), 590-599.

[6] M. Galach, A. Ciechanowska, S. Sabalinska, J. Waniewski, J. Wojcicki and A. Wernynski, Impact of convective transport on dialyzer clearance, J. Artif. Organs 6 (2003), $42-48$.

[7] R.E. Norden and K. Schindhelm, Design of hollow fiber modules for uniform shear elution affinity cell separation, Artif. Organs 21 (1997), 107-115.

[8] T. Osuga, T. Obata, H. Ikehira, S. Tanda, Y. Sasaki and H. Naito, Dialysate pressure isobars in a hollow-fiber dialyzer determined from magnetic resonance imaging and numerical simulation of dialysate flow, Artif. Organs 22 (1998), 907-909.

[9] S. Baigent, M. Williams, A. Marks, M. Penney, J. Wattis, P. Howell, J. King, and J. Chapman, Mathematical modelling of profiled haeomodialysis, 1st Mathematics in Medicine Study Group, Nottingham, 2000.

[10] M. M. Ursino, L. Coli, M. Grilli Ciciloni, V. Dalmastri, A. Guidicissi, P. Masotti, G. Avanzolini, S. Stefoni, and V. Bonomini, A single mathematical model of intradialytic sodium kinetics: in vivo validation during hemodialysis with constant or variable sodium, Int. J. Artif. Organs 19 (1996), no. 6, 393-403.

[11] F. Yan, P. Dejardin, A. Schmitt and C. Pusineri, Electrochemical characterization of a hemodialysis membrane, J. Phys. Chem. 97 (1993), 3824-3828.

[12] L. Coli, M. Ursino, A. De Pascalis, C. Brighenti, V. Dalmastri, G. La Manna, E. Isola, G. Cianciolo, D. Patrono, P. Boni, and S. Stefoni, Evaluation of Intradialytic Solute and Fluid Kinetics: Setting Up a Predictive Mathematical Model, Blood Purif. 18 (2000), $37-49$.

[13] W. Krebs and W. Pickl, A Mathematical Model of Hemodialysis, in Modelling, Analysis, and Optimization of Biosystems, Springer, Berlin, 2007, pp. 141-183.

[14] F. Locatelli, C. Manzoni, and S. Di Filippo, Electrolyte Balancing: Modern Techniques and Outcome, Blood Purif. 19 (2001), 195-199.

[15] Ronald L. Fournier. Basic Transport Phenomena in Biomedical Engineering (2nd Ed.), Taylor \&Francis, 2007.

[16] D.A. Saville. Electrohydrodynamics: The Taylor-Melcher Leaky Dielectric Model, Ann. Rev. Fluid Mech. 29 (1997), 27-64.

[17] K. Yamamoto, M. Hayama, M. Matsuda, T. Yakushiji, M. Fukuda, T. Miyasaka, K. Sakai, Evaluation of asymmetrical structure dialysis membrane by tortuous capillary pore diffusion model, Journal of Membrane Science 287 (2007), 88-93. 